\title{
LXIV. On the reaction of effluent water, and on the maximum effect of machines. With notes relating to the theory of Barker's Mill
}

\author{
Mr. P. Ewart \& J. Ivory Esq. M.A. F.R.S.
}

To cite this article: Mr. P. Ewart \& J. Ivory Esq. M.A. F.R.S. (1828) LXIV. On the reaction of effluent water, and on the maximum effect of machines. With notes relating to the theory of Barker's Mill , Philosophical Magazine Series 2, 3:18, 416-430, DOI: 10.1080/14786442808674679

To link to this article: http://dx.doi.org/10.1080/14786442808674679

进 Published online: 10 Jul 2009.

Submit your article to this journal $\pi$

Ш Article views: 2

View related articles $\sqsubset$ 
colouring painted on either retina, by which the separation of their forms can be distinguished?

Press the axis of either eye sufficiently to the right or left, a larger quantity of colouring will immediately be painted upon one retina than upon the other, which will separate their interior and horizontal edges, and troo images will thence immediately and necessarily arise upon the perception of the mind.

1 feel convinced that the more these ideas are contemplated, and the more clearly they are apprehended, the better will they serve to elicit the reason of several other phænomena concerning vision, which it has hitherto been considered difficult to explain; and what is of still greater importance, they may throw some light upon those which belong to every analogous operation of the human senses and intellect.

LXIV. On the Reaction of efluent Water, and on the Maximum Effect of Machines. By Mr. P. EwarT*. With Notes relating to the Theory of Barker's Mill. By J. Ivonx, Esq., M.A.F.R.S.†

THE following important proposition relating to this subject, is laid down by Daniel Bernoulli in his "Hydrodynamics," page 278. If a jet of water I (fig. 1.) issue from the side of a vessel $A$, with the velocity which a body would acquire in falling freely from the surface $\mathrm{B}$ to $\mathrm{C}$, he says the repulsion of the water in the opposite direction to the jet will be equal to the weight of a column of water, of which the base is equal to the section of the contracted vein, and the height equal to $2 \mathrm{BC}$.

This qucstion respecting the amount of what has been termed the "reaction of the effluent water," derives additional interest from the circumstance of its having particularly engaged the attention of Sir Isaac Newton, and from his having given a

* From a paper " On the measure of moving force," in the Memoirs of the Lit. and Phil. Society of Manchester, second series, vol. ii. 1813.

We insert this extract because it treats, correctly we believe, of subjects which have engaged the attention of many eminent mathematicians (in former times as well as recently), whose reasonings and conclusions on the points in question are at variance with each other. These discrepancies are to be regretted, inasmuch as some of the essential points in the application of the principles of mechanics to practical purposes are involved in them.--Eort.

+ Communicated by the Author.

We are glad to lay before those of our readers who have attended to the various intricate theories that have been offered of the action of Barker's Mill, Mr. Ivory's Notes on that subject.-EDIr. 

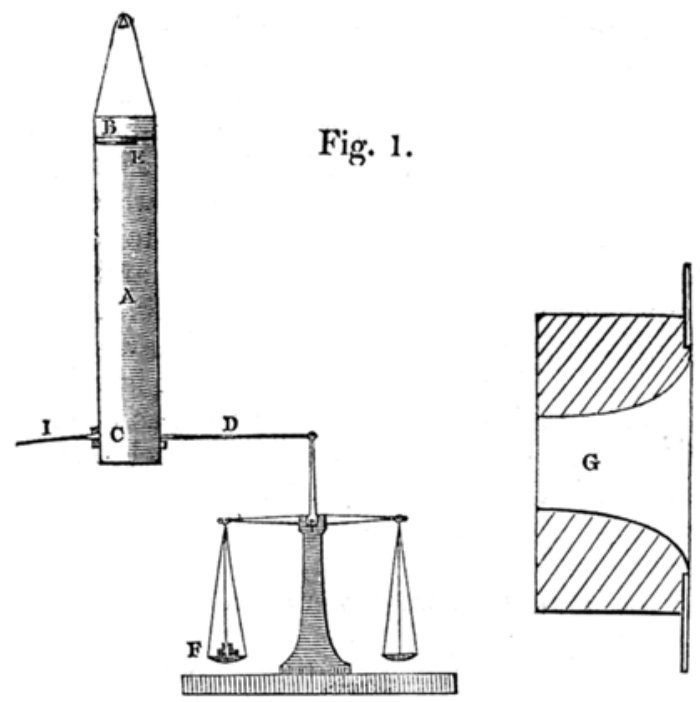

solution of the problem in the first edition of the "Principia," which he materially altered in the succeeding editions. In the first edition (book 2nd, prop. 37.) he infers, that the reaction is equal to the weight of a column of water of which the base is equal to the area of the orifice, and the height equal to that of the surface of the water above the orifice. In the succeeding edition, the subject is more fully discussed in the 36 th prop. of the second book, where he infers (cor. 4.) that, when the area of the surface $B$ is indefinitely large compared with that of the orifice, the reaction is, what it was afterwards in a different manner demonstrated to be by D. Bernoulli. Sir Isaac Newton further observes, that he found, by admeasurement, the area of the orifice in a thin plate to be to that of the section of the contracted vein, at the point of its greatest contraction, in the ratio of $\sqrt{2}: 1$ nearly. He takes the reaction, therefore, to be greater than what he understood it to be when he published the first edition, in the ratio of $\sqrt{2}$ : 1 nearly. He refers, however, more to experiment than to theory for a solution of this question; and many valuable experiments have since been made on effluent water; yet I cannot find that the results of any direct experiments have been published which go to determine the precise amount of this reaction.

Sir Isaac Newton suggested (Principia, first edit. p. 332.) a method by which the reaction may be easily measured. If the vessel be suspended like a pendulum, he observes, it will

Nerw Series. Vol. 3. No. 18. June 1828. $3 \mathrm{H}$ recede 
recede from the perpendicular in the opposite direction to the jet.-I have made some experiments on a vessel suspended in that manner; and in order to ascertain the reaction as accurately as possible, I made use of a balance-beam furnished with a perpendicular arm of the same length as the horizontal arms, as represented at fig. 1 . The scales were exactly balanced, and the end of the rod $\mathrm{D}$ made just to touch the side of the vessel.-The orifice was then opened, and the water in the vessel was kept uniformly at the same height by a stream falling gently on the plate $\mathrm{E}$. The scale $\mathrm{F}$ having been raised by the reaction of the jet, weights were put into it till it was brought exactly to the position in which it was before the orifice was opened. The diameter of the vessel was 7 inches, and the height $\mathrm{BC}$ exactly 3 feet. I tried orifices of various diameters from $\cdot 35$ to $\cdot 7$ of an inch. Their exact diameters were ascertained by a micrometer, and the time carefully observed in which 30 lbs. of water were discharged through each orifice.

When the orifice was made in a thin plate $\left(\frac{1}{30}\right.$ th of an inch in thickness), I found the reaction to be greater than that stated in Sir Isaac Newton's first conclusion, in the ratio of $1 \cdot 14$ to 1 . There was some variation in the results of the experiments. The greatest reaction, however, was as $1 \cdot 16$ to 1 , and the least as 1.09 to 1, which fall far short of Sir Isaac Newton's last inference. The velocity of the water at the orifice (ascertained by observing the time in which 30 lbs. were discharged) was less than that which a body would acquire in falling freely from $B$ to $C$, in the ratio of $\cdot 6$ to 1 .

I found no constant ratio to subsist between the diameter of the contracted vein and that of the orifice; and observing considerable opacity in the jet at the contracted vein, I concluded it to be divided into a number of different filaments, and I gave up all hopes of ascertaining the actual area of the section of the stream at that place by measuring its diameter. After repeated trials I found that when the water issued through a contracted hole, of the shape represented at $G$, the jet was quite transparent, and the reaction (taking the mean of 12 experiments with 4 different orifices) was less than the weight of a column of water of twice the height of the head and diameter of the smallest part of the hole, in the ratio of .865 to 1 . The least reaction was as $\cdot 85$ to 1 , and the greatest as $\cdot 88$ to 1 . By measuring the quantity of water delivered in a given time, I found the velocity of the jet, at the smallest part of the orifice, to be less than that which a body would acquire in falling freely from $\mathrm{B}$ to $\mathrm{C}$, in the ratio of 94 to 1 . The highest ratio was as $\cdot 95$ to 1 , and the lowest $\cdot 89$ to 1 . 
From these results it appears, that when the contracted vein is not opaque, and when its velocity is nearly equal to that which is due to the head, the reaction is nearly equal to what it was concluded to be by Sir Isaac Newton and M. Dan. Bernoulli; and the great apparent difference between Sir Isaac Newton's first and second conclusions arises from his having been misled by some experiments to which he alludes. He says-" Per experimenta vero constat, quod quantitas aquæ, quæ, per foramen circulare in fundo vasis factum, dato tempore effluit, ea sit, quæ cum velocitate prædicta," [viz. the velocity due to the head] " non per foramen illud, sed per foramen circulare, cujus diametrum est ad diametrum foraminis illius ut 21 ad 25 , eodem tempore effluere debet*." We must presume, however, that he refers to experiments made by others; for if he had made them himself, he would, no doubt, have arrived at the same results which have since been so well established by various authors, and he would have stated the above ratio to be as 19.5 to 25 nearly.

But his demonstration of the reaction requires that the velocity at the contracted vein shall be equal to that which is due to the head. Now that velocity cannot be delermined by measuring the imperfectly contracted vein in cases of water spouting through a hole in a thin plate.

We may safely indeed infer, that, in such cases, the velocity is considerably less than what is due to the head. For, the jet being opaque, some moving force must be expended in separating the particles from each other, and the distance to which the jet from such an orifice is projected on a horizontal plane, confirms that inference. The demonstration, therefore, of the reaction, can be properly applied to such cases only as those where the water, issuing through a tube properly contracted, acquires the velocity nearly which is due to the head, and in those cases the experimental results agree, as $I$ have stated, remarkably well with the demonstration.

These results agree also with the explanations which have been given of moving forcet. If we suppose the velocity of the jet to be equal to that which is due to the head, and the vessel to move uniformly in the opposite direction $\mathrm{CD}$ with the same velocity; the water will be at rest as it issues.

Let $a$ represent the area of the smallest section of the orifice. Then while the vessel has moved through a space $=$ $2 \mathrm{BC}$, a quantity of water represented by $a \times 2 \mathrm{BC}$ has de-

* Principia, edit. ii. lib. 9. prop. 36.

+ By moving force is meant the product of the pressure into the space through which it acts, or of the quantity of water into the height through which it falls. The stme sense in which the term is used by Euler. 
scended from $\mathrm{B}$ to $\mathrm{C}$, and has been brought to rest. But the reaction is $=a \times 2 \mathrm{BC}$, and this multiplied by $2 \mathrm{BC}$, the space through which it has acted, gives $a \times \overline{2 \mathrm{BC}}^{2}$ for the amount of the moving force produced, which is exactly the quantity of moving force necessary to raise the column $a \times 2 \mathrm{BC}$ to the height $\mathrm{BC}$, and to project it with the velocity $2 \mathrm{BC}$. For, a moving force $=a \times 2 \mathrm{BC} \times \mathrm{BC}$ will raise that column from $\mathrm{C}$ to $\mathrm{B}$, and an equal moving force will generate the velocity $2 \mathrm{BC}$ in the same column, therefore $2 a \times 2 \mathrm{BC} \times \mathrm{BC}=a \times\left.\overline{2 \mathrm{BC}}\right|^{2}$ is the whole moving force necessary to restore that column to the place and condition in which it was before it began to descend; and as no moving force has been expended in producing change of figure, that quantity of moving force must be found in the reaction of the water through the space which the vessel has moved while the water descended and was brought to rest.

Upon the same principle an easy and simple explanation may be given, I apprehend, of the action of the hydraulic machine called Barker's Mill. Let $\mathrm{AB}$ (fig. 2.) be the perpendicular tube, and BC the horizontal arm; let $v$ express, in feet per second, the rotatory velocity of the arm at the orifice $\mathrm{C}$, and let the water be supposed to issue with the velocity due to the pressure*: Put $g=16_{\frac{1}{12}}$ feet.

If $\mathrm{BC}$ be a cylindrical tube, and if $q$ represent the quantity of water it contains from $\mathrm{B}$ to $\mathrm{C}$, the centrifugal pressure upon a section of the arm at $\mathrm{C}$, will be $\frac{q v^{2}}{4 \mathrm{gBC}}$; and whatever the length $\mathrm{BC}$ may be, the diameter remaining the same, $q$ being as $\mathrm{BC}$, the centrifugal pressure at $\mathrm{C}$ will always be as $\tau^{2}$; and it will be equal to the pressure of a perpendicular column of water whose height in feet is $\frac{v^{2}}{4 g}$. Then if $h$ express in feet the height $\mathrm{AB}$ of the water in the vertical tube, $h+\frac{v^{2}}{4 g}$ will be the whole pressure at $\mathrm{C}$; and if $a$ express in feet the area of the most contracted section of the orifice, $2 a\left(h+\frac{v^{2}}{4 g}\right)$ will

* It is here understood that the areas of the sections of the perpendicular tube and of the horizontal arm shall be indefinitely large when compared with the area of the orifice. 
express the reaction, which being multiplied by $v$, the space through which it acts in a second, gives $2 a v\left(h+\frac{v^{2}}{4 g}\right)$ for the total moving force of the arm in a second. But a part of this moving force is expended in producing the rotatory motion of the water, and in raising it to the height $\frac{v^{2}}{4 g}$. For, if we suppose a perpendicular tube $\mathbf{C P}$ to rise from the arm at $C$, the surface of the water in that tube would stand at P, PR being $=\frac{v^{2}}{4 g}$. Now if instead of letting the water escape at $C$, it be allowed to flow over the perpendicular tube at $P$, and fill another similar perpendicular tube adjoining it, and issue from an orifice at the bottom of that tube, the effect must be the same as if it issued at $\mathbf{C}$, and a moving force must be expended at $\mathbf{C}$, sufficient to generate the velocity $v$, in the water which passes, and also to raise it from $R$ to $P$.

The pressure at $\mathbf{C}$ being equal to the weight of a column of water whose height is $h+\frac{v^{2}}{4 g}$, (that is $=\mathrm{AB}+\mathrm{PR}$ ), the velocity with which the water issues will be $\sqrt{4 g\left(h+\frac{v^{2}}{4 g}\right)}$ or $\sqrt{4 g h+v^{3}}$. Let $\mathrm{V}$ express that velocity, then $a \mathrm{~V}$ will express the quantity of water which passes in a second; and $2 a \mathrm{~V} \frac{v^{2}}{4 g}$ will express the moving force necessary to generate the velocity $v$, in that quantity of water, and to raise it from $\mathbf{R}$ to $\mathbf{P}$. That quantity of moving force being deducted from the total moving force of the arm, leaves $2 a v\left(h+\frac{v^{2}}{4 g}\right)$ $-2 a \mathrm{~V} \frac{\boldsymbol{v}^{2}}{4 g}$ for the effective moving force of the arm in a second.

That this is the effective moving force, may be shown also in another manner, as follows :

The absolute velocity of the water after it has left the machine will be $\mathrm{V}-v$, and $\frac{(\mathrm{V}-v)^{2}}{4 g}$ will be the head which would produce that velocity; which being multiplied by $a \mathrm{~V}$, the quantity of water delivered in a second, gives $a \mathrm{~V} \frac{(\mathrm{V}-v)^{2}}{4 g}$ for the moving force which remains with the water after it has left the machine.

If that be deducted from $a \mathrm{Vh}$, the whole moving force of the water, there will remain $a \mathrm{~V} h-a \mathrm{~V} \frac{(\mathrm{V}-v)}{4 g}$ for the effective moving force, which will be found to be equal to $2 a v\left(h+\frac{v^{\circ}}{4 g}\right)$ $-2 a \mathrm{~V} \frac{v^{2}}{4 g}$, the affective moving force stated above. 
The theory of this machine has occasionally occupied the attention of many distinguished mathematicians, and M. Euler has given two elaborate treatises on its principles in the Memoirs of the Berlin Academy for 1750, p. 311 ; and for 1751, p. 271. His demonstrations relating to this subject are very complicated, and they do not appear to have been adopted by succeeding authors*.

Mr. Waring, of America, has given quite a different theory, which has been approved of by several good writers on hydraulics. He concludes that the greatest effect will be produced when the velocity of the orifice is half that of the issuing water; and that this effect will be nearly the same as that of a wellconstructed undershot water-wheel + .

The explanation which I have offered of the action of the water on this machine is different from any other that $I$ have had an opportunity of consulting. I offer it, therefore, merely as an attempt to solve an intricate problem.

If it were possible for the water to issue with the velocity due to the pressure, it is obvious, if my explanation be right, that although a very large proportion of the moving force of the water may be communicated to the machine, moving with a moderate velocity, the maximum of effect can only be obtained by an infinite velocity. But when the water issues with a velocity which is less than what is due to the pressure, as must always be the case in practice, the velocity at which the maximum of effect is produced, may be found as follows. It should first be ascertained by experiment how near the issuing velocity can be brought to that which is due to the pressure. From the experiments which $I$ have made, $I$ have been led to conclude that no greater issuing velocity can possibly be obtained from a machine of this kind than what is due to 8 of the pressure. If this conclusion be correct, it follows, that, whatever may be the issuing velocity of the water, a moving force, equal to $\frac{1}{4}$ of the moving force which is necessary to generate that velocity in the water, when falling freely, is expended in producing change of figure; that is, in forcing the water through the tubes and through the orifice $\mathrm{C}$; and if the velocity of the machine be such that $\mathrm{PC}=5 \mathrm{AB}$, the issuing velocity will be equal to the velocity of the orifice, and the whole moving force of the water in descending from $A$ to $B$ will be expended in producing change of figure.

For, the head due to $\mathrm{V}$, the issuing velocity, will in this case

* M. Euler says, "In employing the same quantity of water, and the same fall, this machine will produce an effect nearly four times greatcr than the ordinary machines."

† American Philos. Trans. vol. iii. p. 191 and 199. 
be PR, which is also the head due to $v$, the velocity of the orifice. We shall therefore have $\mathrm{V}=v$; and if $\mathrm{CP}$ represent the total moving force necessary to raise the water from $\mathrm{C}$ to $P, C R=A B$ will represent that part of it which is expended in producing change of figure. The greatest velocity, therefore, that the orifice, when the machine meets with no resistance, can acquire, will be $\sqrt{4 g \times 4 h}$.

When the velocity of the orifice is less than that, $V$ will be greater than $v$; and $\mathrm{V}-v$, the absolute velocity of the water after it has left the machine, will be $\sqrt{\cdot 8\left(4 g h+v^{2}\right)}-v$. The head or the moving force expended in producing that velocity will be as $\frac{\sqrt{\left.\frac{.8\left(4 g h+v^{2}\right)}{4 g}\right)^{2}}}{4 g}$.

The moving force expended in producing change of figure will be as $2\left(h+\frac{v^{2}}{4 g}\right)$. Now when the sum of these two quantities, or $\frac{\sqrt{\sqrt{\cdot 8\left(4 g h+v^{2}\right)}-v}}{4 g}+\cdot 2\left(h+\frac{v^{2}}{4 g}\right)$, is a minimum, we shall find $v=\sqrt{2 g h(\sqrt{5}-1)}=6.3056 \sqrt{h}$ for the velocity of the orifice when the machine produces a maximum of effect; and in that case the above sum becomes $=\cdot 4.42 h$.

We shall therefore have $h-\cdot 4472 h=\cdot 5528 h$ for the maximum of effect, supposing $h$ to represent the whole moving force of a given quantity of water descending from $A$ to $B$. This effect is considerably greater than that which the same quantity of water would produce if applied to an undershot water-wheel, but less than that which it would produce if properly applied to an overshot water-wheel.

Respecting the maximum of effect produced by machines, I wish to observe, that in the actual construction of machines it is necessary to aim at a maximum quite different from that which is usually proposed in books on the theory of mechanics. This will perhaps be best explained by examining the simple case where a given weight $\mathrm{P}$, (fig. 3) connected with another $\mathrm{W}$, by a string passing over the pulley $\mathrm{F}$, descends vertically and raises $W$, without friction, from the horizontal line $\mathrm{AC}$ along the inclined plane $\mathrm{AB}$. If we

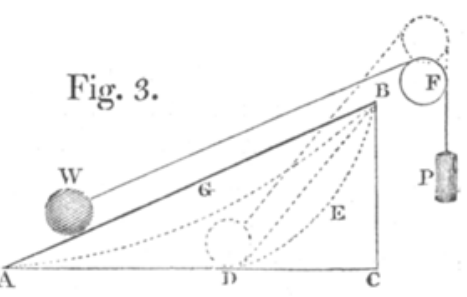
make $\mathrm{AB}: \mathrm{BC}:: 2 \mathrm{~W}: \mathrm{P}, \mathrm{W}$ will be raised to $\mathrm{B}$ in the least time; 
time*; and upon this principle, the maximum of effect in machines is usually demonstrated in theory. In practice, however, the object is not merely to raise $W$ to $B$ in the least time, but to raise it with the least expenditure of moving force. When it is raised in the least time, $P$ must descend through a space $=A B$, but when it is raised with the least moving force, $\mathrm{P}$ descends through a space $=\frac{1}{2} \mathrm{AB}$ only. For, if we make $\mathrm{BD}=\frac{1}{2} \mathrm{AB}$, and let $\mathrm{W}$ ascend along any concave surface $\mathrm{DEB}$, of which $\mathrm{BD}$ is the chord, it will be raised to $\mathrm{B}$ by the descent of $P$ through a space $=B D$, and it will be at rest when it arrives at $B$. This is so obvious, that it would be superfluous to give a demonstration of it. It appears then, that twice the quantity of moving force which is absolutely necessary to raise $W$ to $B$, must be expended if it is to be raised by $P$ in the least time. To determine the curve by which $W$ will ascend from $D$ to $B$ in the least time, is an intricate problem, and I do not know that it has ever been solved +; but a practical approximation to it in any particular case may be easily found. A well constructed steam-engine for raising water exhibits in every stroke a practical example of the same problem. At the commencement of the stroke, a very great pressure of steam is thrown upon the piston, and this pressure is gradually diminished, so that at the end of the stroke there is a considerable preponderance in the opposite direction. In consequence of this regulated pressure of the steam, the motion of the machine resembles the uniform vibrations of a pendulum, and the moving force of the steam is applied to the greatest advantage.

By proceeding on the principle that when $W$ is raised to $B$ in the least time, the maximum of effect is produced, many erroneous conclusions have been drawn respecting the proper construction of machines. It is laid down for example, on this principle, that "In an overshot water-wheel, the machine will be in its greatest perfection, when the diameter of the wheel is two-thirds of the height of the water above the lowest point of the wheel $\ddagger . "$ But it is very well known that there would be lost, by that construction, nearly one-third of the moving force of the water, which is saved by making the wheel one-half larger in diameter, and by making its velocity much less than what is required by the above rule.

* If the ascent be made in the least possible time, $\mathrm{W}$ must ascend not along the plane $\mathrm{AB}$, but along a concave surface $\mathrm{AGB}$.

$\uparrow$ This difficult problem, we understand, has lately been solved by the Rev. E. Sibson of Ashton, in Makerfield, Lancashire, and the solution will appear in the next volume of the Memoirs of the Lit. and Phil. Society of Manchester.-Eort.

† Gregory's Mechanics, vol, i. p. 447. 
Notes relating to the Theory of Barker's Mill. By J. Ivory, Esq. M.A. F.R.S.

\section{On the Pressure caused by the Centrifugal Force.}

The centrifugal pressure may be investigated in the manner following.-The whole pressure at the orifice is the sum of the variable pressures of all the molecules, or infinitely small portions of the fluid, in the length of the arm. Let $r$ be the length of the arm between the oritice and the axis; $g=16_{\frac{1}{12}}^{1}$ feet; $v$, the absolute velocity of the orifice in feet: then if $d x$ (or $x$ ) be a small portion of the fluid in the arm at the distance $x$ from the axis, the centrifugal pressure of $d x$ will be

$$
\frac{x^{2} v^{2}}{r^{2}} \times \frac{d x}{2 x g}=\frac{v^{2} \cdot x d x}{2 g r^{2}}
$$

and the pressure of the prism of the fluid, of which the length is $x$, will be $=\frac{v^{2} x^{2}}{4 g r^{2}}$; and the whole pressure of all the fluid in the length $r$, will be $=\frac{v^{2} r^{2}}{4 g r^{2}}=\frac{v^{2}}{4 g^{2}}$.

The same thing may be more shortly stated, thus: The centrifugal force of every small portion of the fluid in the arm being as the distance from the axis, we may assume that every portion is acted upon by the force which takes place at the middle of the arm, or at the distance $\frac{1}{2} r$ from the axis. Now this force is $=\frac{v^{2}}{4 g r}$; which multiplied by $r$, the sum of the portions of the fluid, gives $\frac{v^{2}}{4 g r} \times r=\frac{v^{2}}{4 g}$, the same as be-
fore.

And since we have,

$$
\frac{v^{2}}{4 g}=\frac{r^{2}}{4 g} \times\left(\frac{v}{r}\right)^{2}
$$

$\frac{v}{r}$ being the angular velocity of the machine, or the angle through which it turns in a second; it follows that the angular velocity being the same, the centrifugal pressure varies as the square of the length of the arm.

Some authors, and in particular Bossut (Hydrod. tom. i. $\$ 4.29$, reckon the centrifugal pressure, not in the whole length $r$, but in the distance between the upright tube and the orifice in the horizontal arm. Let $r^{\prime}$ be the radius of the up-

New Series. Vol. 3. No. 18. June 1828. 3 I right 
right tube; then, according to the authors mentioned, the centrifugal pressure at the orifice will be equal to

$$
\frac{v^{2}}{4 g} \times \frac{r^{2}-r^{\prime 2}}{r^{2}}
$$

being the difference between the pressures at the clistances $r$ and $r$, from the axis.

II. Velocity with which the Fluid issues from the horizontal Arm, supposing that $v$ is the absolute Velocity of the Orifice.

The velocity required is produced by the pressure of the fluid in the upright tube increased by the centrifugal pressure. Wherefore if $h$ denote the length of the upright tube, supposed to be kept constantly full, the sum of the two pressures mentioned will be $=h+\frac{v^{2}}{4^{g}}$; and, if $\mathrm{V}$ denote the velocity of the effluent water, we shall have,

And, if we put $v^{2}=4 g f$, then

$$
\mathrm{V}^{2}=4 g\left(h+\frac{m^{2}}{4 g}\right)=4 g h+v^{2} .
$$

$$
\mathrm{V}^{2}=4 g(h+f) \text {. }
$$

III. Demonstration of Daniel Bernoull's Proposition respecting the Reaction of effluent Water (or, Cor. 2. prop. 36. lib. 2. Principia).

Suppose that water issues from a small orifice in the side, or bottom, of a vessel which is kept full; let $k$ be the height of the surface above the level of the orifice: then, $2 \sqrt{g k}$ is the velocity in a second with which the water issues; and, if $a$ be the area of the orifice, the quantity of water discharged in a second is equal to the prism $a \times 2 \sqrt{g k}$; and, as the water issues with the velocity $2 \sqrt{g k}$, the quantity of motion in the water discharged in a second is equal to,

$$
a \times 2 \sqrt{g k} \times 2 \sqrt{g h}=2 a k \times 2 g ;
$$

and the same quantity of motion; viz. $2 a k \times 2 g$, is evidently the reaction of the water projected in a second. But the prism or weight, $2 a k$, by falling, produces a quantity of motion equal to $2 a k \times 2 g$ in a second: wherefore the reaction of the projected fluid is equal to the weight $2 a k$; that is, to the wejght of a prism of the fluid having its base equal to the orifice $a$, and its altitude equal to $2 k$.

This proposition supposes that the water has acquired its complete velocity of projection, due to the head $k$. Before the 
efflux commences, and while the fluid is at rest, the weight $a k$ is sufficient to counteract the tendency to begin motion.

IV. Computation of the Moving Force of Barker's Mill, or of the impulse produced by the Reaction of the projected Water; supposing that $h$ is the length of the upright Tube kept constantly, full, and $v$ the absolute Velocity of the Orifices in the horizontal Arms.

This machine has generally two horizontal arms diametrically opposite; but it may have any number of such arms with an orifice in each. Whatever be the number of the arms, I shall suppose that all the orifices are at the same distance, $r$, from the axis; and I shall use the symbol $a$ to denote the sum of the areas of all the orifices.

The velocity $V$ with which the water issues from the orifices is known by the formula,

$$
\mathrm{V}^{2}=4 g h+v^{2}=4 g(h+f) .
$$

And, since the water is propelled from the orifices with the velocity $\mathrm{V}$, and has acquired from the machine the velocity $v$ in the opposite direction, the velocity with which it is projected from the arms, is equal to $\mathrm{V}-v$ : but $a \mathrm{~V}$ is the quantity of water so projected in a second; wherefore the momentum of the projected water is equal to $a \mathrm{~V} \times(\mathrm{V}-v)$; and the same expression is equal to the impulse communicated to the machine by reaction. Wherefore, since the impulse is exerted at the end of the lever $r$, its effect to turn the machine, or the motive force, is equal to

$$
r \times a \mathrm{~V} \times(\mathrm{V}-v) .
$$

We may likewise measure the motive force by the momentary impulse multiplied by the space through which it acts; and the force accelerating the machine will be,

$$
v \times a \mathrm{~V} \times(\mathrm{V}-\mathrm{v}) \text {. }
$$

These two formulæ seem to be the most elementary expressions of the motive force of this machine.

$$
\text { Mr. Evart's Formula. }
$$

$$
\text { Let us put } \quad \frac{a \mathbf{V} \times(\mathbf{V}-v)}{2 g}=\mathrm{P} \text {; }
$$

then, $a \mathrm{~V} \times(\mathrm{V}-v)=\mathrm{P} \times 2 \mathrm{~g}$; wherefore the reaction is equal to the momentum which the weight $P$ acquires in a second by falling; in other words, the momentary impulse of reaction is equal to the pressure of the weight $\mathbf{P}$. The mo- 
tive force of the machine, according to the formula (2), is therefore equal to $\mathrm{P} \times v$. Now

$$
\mathrm{P} \times v=2 a v \times \frac{\mathrm{V}^{2}}{4 g}-2 a \mathrm{~V} \times \frac{v^{2}}{4 g}
$$

and, by substituting the value of $\mathrm{V}^{2}$,

$$
\mathrm{P} \times v=2 a v\left(h \times \frac{v^{2}}{4 g}\right)-2 a \mathrm{~V} \frac{v^{2}}{4 \mathrm{~g}} \text {. }
$$

Euler's Formula.

In the formula (1), put $2 \sqrt{g(h+f)}$ and $2 \sqrt{g f}$ for $\mathrm{V}$ and $v$; then the impulse to turn the machine will be,

$$
2 a r \times 2 g\left(h+f-\sqrt{h f+f^{2}}\right),
$$

which is Euler's formula.

N. B. It must be observed that Fuler makes gravity the unit of forces; that is, he denotes it by 1 , and expresses the velocities by the square roots of the heights : and, in order to make the foregoing expression agree with Euler's assumption, we must make $2 g=1$; because $2 g$ has been taken for the measure of the force of gravity.

\section{Bossut's Formula.}

This author considers the machine in a form more complicated than we have here contemplated; but, when we make allowance for the peculiar mechanism he supposes, the result of his investigation will be found to agree with the expression (1).

\section{Effect of the Machine.}

We have found that the moving power of the machine is $\mathrm{P} \times v$; and

$$
\mathrm{P} \times v=v \times \frac{a \mathrm{~V} \times(\mathrm{V}-v)}{2 g}=a \mathrm{~V} \times \frac{v^{2}}{2 g} \times\left(\frac{\mathrm{V}}{v}-\mathrm{l}\right) .
$$

Now $a \times \mathrm{V}$ is the prism of water that issues from the orifices in a second, which quantity we shall denote by $Q$; then the equation,

$$
\mathrm{Q}=a \times \mathrm{V}
$$

will express the relation between the water expended, the velocity of expenditure, and the sum of the areas of the orifices. Further, put

$$
x=\frac{v}{\mathrm{v}}=\frac{\sqrt{f}}{\sqrt{h+f}}
$$


and $x$ will be a number less than 1 : then

$$
\begin{gathered}
f=\frac{x^{2} h}{1-x^{2}}, \\
\frac{v^{2}}{2 g}=2 f=\frac{2 x^{2} h}{1-x^{2}}, \\
\frac{\mathrm{V}}{v}-1=\frac{1-x}{x} ;
\end{gathered}
$$

wherefore, by substitution,

$$
\mathrm{P} \times v=a \mathrm{~V} \times \frac{v^{2}}{2 g} \times\left(\frac{\mathrm{V}}{v}-1\right)=\mathrm{Q} h \times \frac{2 x}{1+x} .
$$

If we make $x=1$, which supposes that $f$ and $v$ are infinitely great, then $\mathrm{P} \times v=\mathrm{Q} h$; and the effect of the machine would be equal to the whole mechanic power in the quantity of water $Q$ falling from the head $h$. This is an unattainable limit; but the nearer $x$ is to 1 , the greater will be the moving force of the machine; which seems to be the only general rule we can have to guide us in the construction of this machine. Having pitched upon the most convenient value of $x$; then,

$$
\begin{aligned}
\mathrm{V} & =\frac{2 \sqrt{g h}}{\sqrt{1-x^{2}}} \\
v & =2 \sqrt{g h} \times \frac{x}{\sqrt{1-x^{2}}}, \\
a & =\frac{\mathrm{Q}}{2 \sqrt{g h}} \times \sqrt{1-x^{2}},
\end{aligned}
$$

effect of the machine, $\mathrm{P} \times v=\mathrm{Q} h \times \frac{2 x}{1+x}$. If $x=\frac{1}{2}$, that is, if $\mathrm{V}$ be double of $v$, then the effect of the machine is $\frac{2}{3} \times \mathrm{Qh}$. In what goes before we have taken the full velocity due to the head, which is always greater than the real velocity in practice. But although the real velocities are less than according to theory, yet they still nearly follow the same proportion ; that is, their squares are as the pressures, or as the heights of the head. We may therefore assume

$$
\mathrm{V}^{2}=4 g \cdot m(h+f),
$$

$m$ being a quantity less than 1 , to be determined experimentally. It is evident that this assumption does not affect the equation (A). We shall now have,

$$
\begin{aligned}
& v=2 \sqrt{g f}, \\
& \nabla=2 \sqrt{g m(h+f)}, \\
& x=\frac{v}{\mathrm{v}}=\frac{\sqrt{f}}{\sqrt{m(h+f)}},
\end{aligned}
$$


4:30 Mr. Ivory's Notes relating to the Theory of Barker's Mill.

$$
\begin{aligned}
\frac{v^{2}}{2 g} & =2 f=2 h \times \frac{m x^{2}}{1-m x^{2}} \\
\mathrm{P} \times v & =a \mathrm{~V} \times \frac{v^{2}}{2 g} \times\left(\frac{\mathrm{V}}{v}-1\right)=\mathrm{Q} h \times \frac{2 m\left(x-x^{2}\right)}{1-n x^{2}} .
\end{aligned}
$$

Now it is obvious that the expression $\frac{x-x^{2}}{1-m x^{2}}$ is susceptible of a maximum; and, by applying the usual rule, the value of $x$ answering to the maximum will be found; viz. $x=\frac{1-\sqrt{1-m}}{m}$. This expression shows that, whatever fraction of unit $m$ stands for, $x$ is contained between the limits $\frac{1}{2}$ and $1^{*}$; so that, when the machine works to the greatest alvantage, $v$ is greater than $\frac{1}{2} \mathrm{~V}$ and less than V. With a given value of $m$, the rules for constructing the machine so as to work to the greatest effect, are contained in the following formulas, in which $n=\frac{1}{m}$, viz.

$$
\begin{aligned}
& x=\frac{1-\sqrt{1-m}}{m}, \\
& v=2 \sqrt{g h} \times \frac{x}{\sqrt{n-x^{2}}}, \\
& \mathrm{~V}=2 \sqrt{g h} \times \frac{1}{\sqrt{n-x^{2}}}, \\
& a=\frac{\mathrm{Q}}{2 \sqrt{g h}} \times \sqrt{n-x^{2}},
\end{aligned}
$$

effect of the machine, $\mathbf{P} \times v=\mathbf{Q} h \times(1-\sqrt{1-m})+$. It now appears that, $m$ falling short of 1 , the effect of the machine decreases rapidly. If $m=\cdot 8$, then $\mathrm{P} \times v=\mathrm{Q} h \times 0.5528$, or $\mathrm{P} \times v=\frac{5}{9} \times \mathrm{Q} h$, nearly.

If $m=\frac{1}{2}$, that is, if half the pressure were lost in forcing the water through the tubes, the effect of the machine would be reduced to $\mathrm{Q} h \times 0 \times 293$, or nearly $\frac{3}{10} \times \mathrm{Q} h$.

$$
x=\frac{1-\sqrt{1-m}}{m}=\frac{1}{2}+\frac{m}{8}+\frac{m^{2}}{16}+\frac{5 m^{3}}{128}+\& c .
$$

when $m=0, x=\frac{1}{2}$; when $m=1, x=1$.

$$
\begin{gathered}
x=\frac{1-\sqrt{1-m}}{m}, \\
m x^{2}=2 x-1, \\
1-m x^{2}=2(1-x) \\
\frac{2 m\left(x-x^{2}\right)}{1-m x^{2}}=\frac{2 m\left(x-x^{2}\right)}{2(1-x)}=m x=1-\sqrt{1-m .}
\end{gathered}
$$

LXV. On 